\title{
A 3-5 GHz Frequency-Tunable Receiver Frontend for Multiband Applications
}

\author{
Chong-Ru Wu, Hsieh-Hung Hsieh, Student Member, IEEE, Li-Shin Lai, and Liang-Hung Lu, Member, IEEE
}

\begin{abstract}
In this letter, a fully integrated receiver frontend which is composed of a low-noise amplifier and a down-conversion mixer is presented for multiband applications. Different from conventional concurrent or switched-type architectures, the proposed circuit uses varactors for the frequency tuning. In addition, broadband techniques are also employed to maximize the operating frequency range and to enhance the circuit performance. Using a standard 0.18 $\mu \mathrm{m}$ CMOS technology, a 3-5 GHz receiver frontend is implemented for demonstration. With a LO power of $3 \mathrm{dBm}$ and an IF frequency of $10 \mathrm{MHz}$, the circuit exhibits a conversion gain of $22.2 \pm 2.4 \mathrm{~dB}$ while the IIP3 and noise figure are better than $-6 \mathrm{dBm}$ and $17 \mathrm{~dB}$, respectively, within the entire frequency range. Operated at a supply voltage of $2 \mathrm{~V}$, the receiver consumes a de power of $16 \mathrm{~mW}$.
\end{abstract}

Index Terms-Band selection, CMOS radio frequency (RF), down-conversion mixer, frequency tuning, low-noise amplifier (LNA), multistandard, receiver frontend.

\section{INTRODUCTION}

D UE to the strong demands on the telecommunication industry, various emerging services such as wireless local networks (WLAN), global positioning systems (GPS) and cellular phones have been deployed with an unprecedented speed in the past few years. In the pursuit of higher data rate, wider bandwidth and more versatile services, new application standards are expected in the near future. As a result, the concept of multiband and multistandard transceivers is introduced to alleviate the hardware compatibility issues among different wireless standards. By integrating the transceivers monolithically and sharing as many of the building blocks as possible, the radio frequency (RF) systems can provide enhanced functionality and flexibility for the users to access various wireless services efficiently.

Until now, various approaches for multiband RF frontends have been proposed including the concurrent and the switched-type methods [1], [2]. Unfortunately, these techniques require additional passive components, resulting in a significant increase in the chip area and hence the fabrication cost. Besides, once the switches are employed, the circuit only allows band selection at discrete frequencies which is not attractive for spectrum saving. In order to overcome the design limitations, a novel receiver frontend topology is presented in this letter

Manuscript received February 2, 2008; revised May 9, 2008. Published September 5, 2008. This work was supported in part by the National Science Council of Taiwan, R.O.C., under Grant 95-2221-E-002-437 and 95-2220-E-002-018 and by the National Chip Implementation Center (CIC).

The authors are with the Graduate Institute of Communication Engineering, National Taiwan University, Taipei 10617, Taiwan, R.O.C. (e-mail: lhlu@cc.ee. ntu.edu.tw).

Digital Object Identifier 10.1109/LMWC.2008.2002491

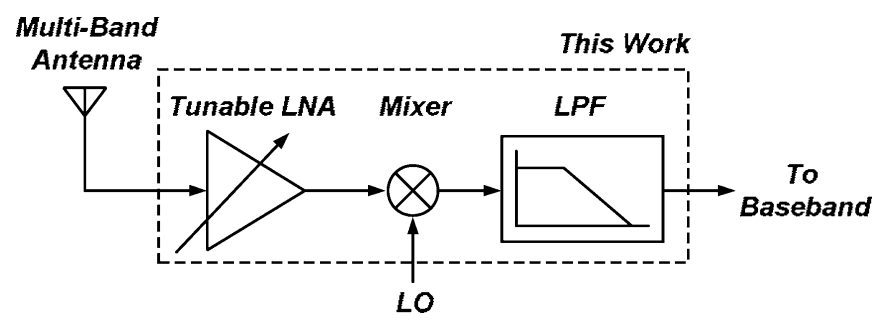

Fig. 1. Proposed multiband receiver frontend.

for multiband operations. By utilizing varactors for the tunable $L C$-load, continuous tuning capability of the center frequency from 3 to $5 \mathrm{GHz}$ is achieved in this particular design. With a compact chip area, the proposed frontend is well suited for the implementation of low-cost multistandard RF receivers for personal wireless communication systems.

\section{PROPOSED TOPOLOGY}

Fig. 1 shows the architecture of the proposed receiver frontend which consists of a low-noise amplifier (LNA), a downconversion mixer and a low-pass filter. In order to provide a wide frequency range for the RF input, broadband techniques are adopted in the circuit design. On the other hand, the multiband operations are achieved by the tunable load of the LNA. For better understanding of the proposed technique, the circuit topology and the design considerations of the LNA and the mixer are described separately as follows.

\section{A. Tunable LNA}

For a multiband LNA design, it is desirable to have a narrowband response for the individual bands such that the out-of-band noise can be suppressed and the output saturation due to the undesirable blockers can be prevented. The most straight-forward approach is to integrate multiple sets of amplifiers in parallel for the bands of interest. However, the resulting hardware complexity and the implementation cost are not practical for a low-cost RF system. In the proposed multiband frontend, the LNA is designed to exhibit broadband impedance matching at the input while a tunable $L C$-load at the output is employed to provide the required narrow-band amplification. The schematic of the proposed LNA is demonstrated in Fig. 2.

1) Broadband Input Matching: As indicated in Fig. 2, the broadband impedance matching at the LNA input is achieved by the fully integrated $L C$-ladder. For better design flexibility, an inductor $L_{3}$ in series with the gate terminal is adopted. Meanwhile, a transformer, which is represented by $L_{1}$ and $L_{2}$ with a mutual inductance $M$, is utilized to reduce the required area and to enhance the amplifier linearity. 


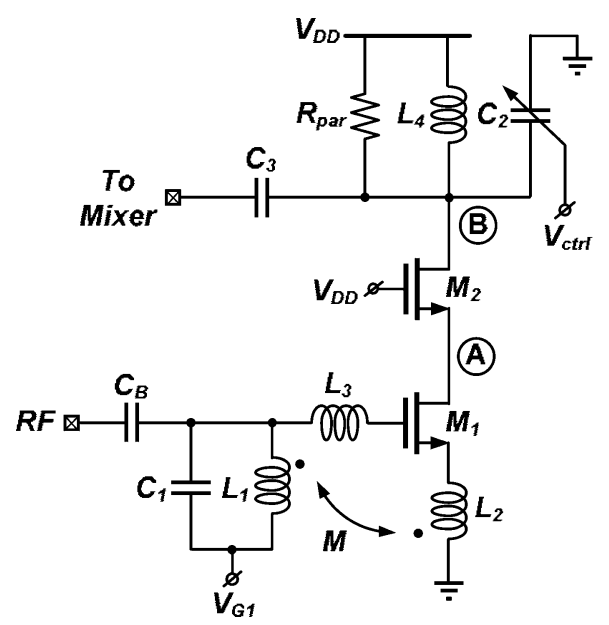

(A)

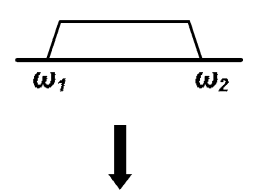

(B)

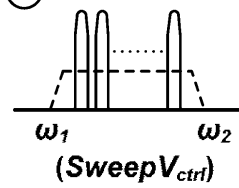

Fig. 2. The proposed LNA for multiband applications.

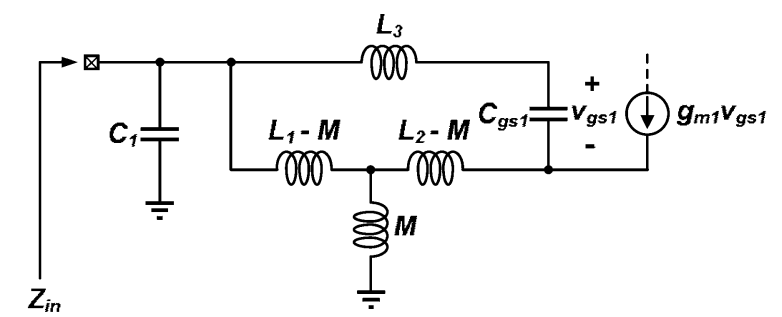

(a)

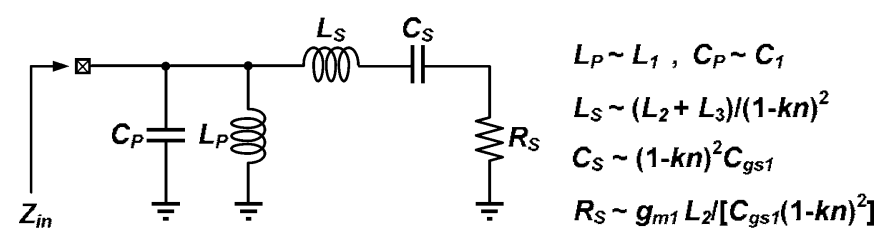

(b)

Fig. 3. (a) The input equivalent circuit of the proposed LNA. (b) The simplified form from (a).

In order to analyze the input matching of the LNA, the small-signal equivalent circuit is shown in Fig. 3(a) where the transformer is modeled by the T-model. By deriving the input impedance $Z_{\text {in }}$, a simplified model as shown in Fig. 3(b) can be used to represent the input network. Note that the circuit parameters are also given in this figure where $k$ and $n$ are the coupling factor and turns ratio of the transformer, respectively. From Fig. 3(b), it is clear that the LNA input can be treated as a resistance-terminated first-order $L C$ bandpass filter. With the design methodology as presented in [3], a wideband impedance matching with maximum RF power transfer can be realized in the proposed LNA topology.

2) Tunable LC Load for Band Selection: Due to the use of the matching technique, the LNA exhibits broadband input characteristics. The transconductance of $M_{1}$ effectively transfers the $\mathrm{RF}$ signal from a voltage form to a current one, which is then injected to the tunable load $\left(L_{4}\right.$ and $\left.C_{2}\right)$ through the cascode stage $M_{2}$. As indicated in Fig. 2, with the parallel $R L C$ network, the output of the LNA demonstrates bandpass characteristics while the passband frequency $\omega_{0}$ is expressed as $\left(L_{4} C_{2}\right)^{-1 / 2}$. Hence, the out-of-band noise and signals at the input are suppressed and

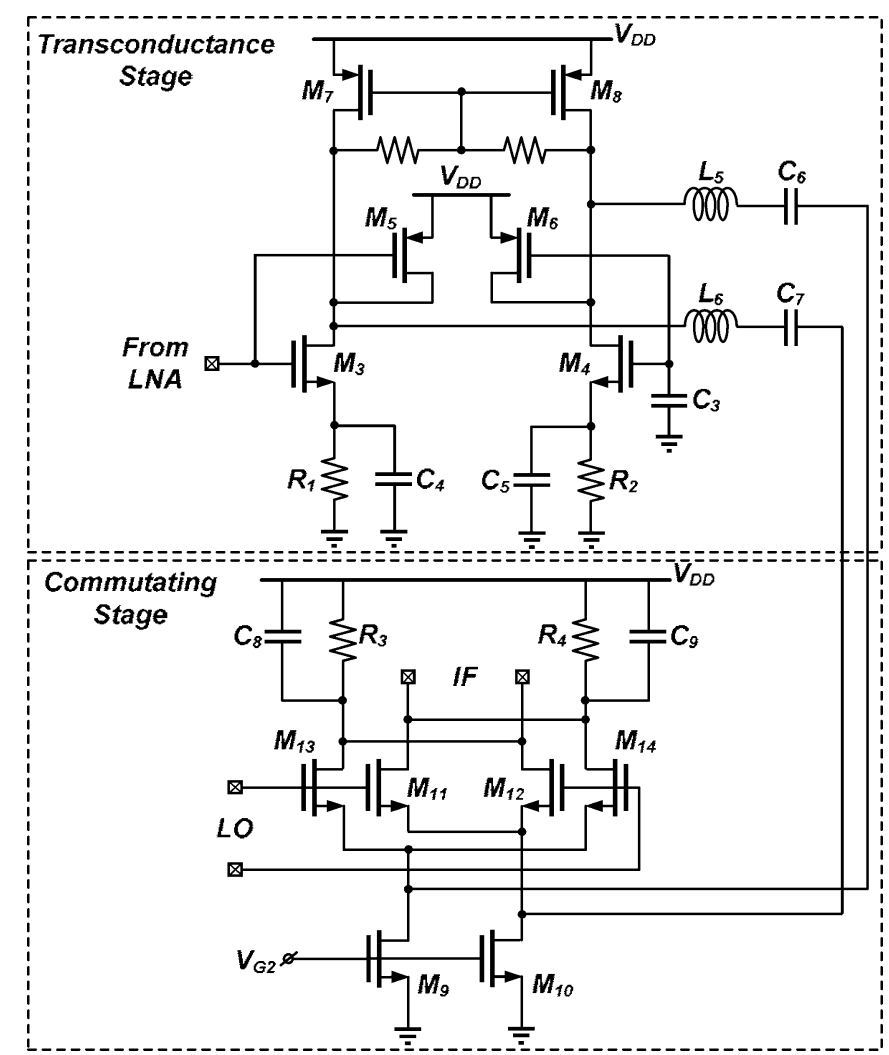

Fig. 4. The proposed topology of the down-conversion mixer.

a narrow-band response is achieved. Note that, in this particular design, the capacitance $C_{2}$ can be varied by the controlled voltage $V_{\text {ctrl }}$, leading to a continuous tuning of the center frequency for the required band selection. Since the tuning mechanism is placed at the output of LNA rather than at input terminal, the noise contribution from the varactor is thus minimized.

\section{B. Down-Conversion Mixer}

The proposed topology of down-conversion mixer is illustrated in Fig. 4. In order to achieve high linearity and low noise figure simultaneously, the dc bias currents of the transconductance and the commutating stages are provided separately. In addition, the current-bleeding technique is also introduced to improve the conversion gain with low power consumption [4]. For a wide input range in the multiband operations, broadband characteristics of the down-conversion mixer are also required. In the proposed circuit topology, the capacitive degeneration is used to compensate the gain roll-off at the higher frequency bands [5]. Meanwhile, inductors $L_{5}$ and $L_{6}$ are inserted between the transconductance and the commutating stages, forming a $L C$-ladder architecture for wideband operations [6]. As for the IF load, a parallel $R C$ connection is employed to provide a lowpass response, leading to better isolation between the LO and IF port.

\section{EXPERIMENTAL RESULTS}

The proposed receiver frontend is implemented in a $1 \mathrm{P} 6 \mathrm{M}$ $0.18 \mu \mathrm{m}$ CMOS process. Fig. 5 shows the microphotograph of the fabricated circuit with a chip area of $1.2 \times 0.95 \mathrm{~mm}^{2}$ including the pad frames. The performance of the receiver 


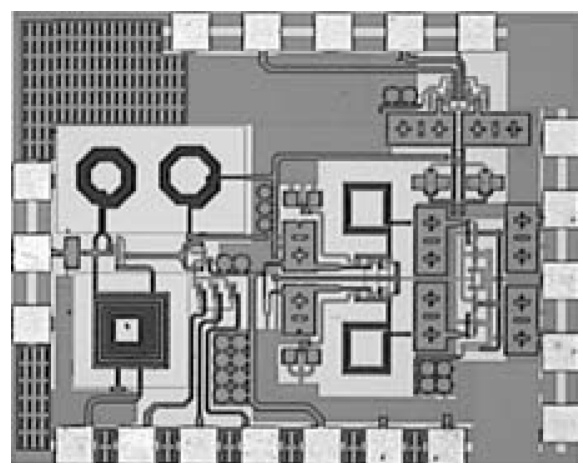

Fig. 5. Die photo of the fabricated receiver front end.

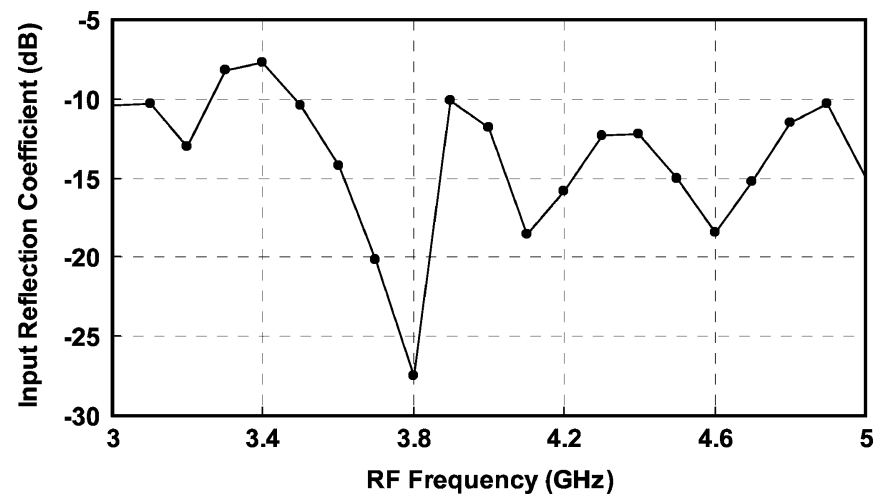

Fig. 6. Measured input reflection coefficient of the front end.

frontend is characterized through on-wafer probing while the losses accounted for the measurement setup were calibrated and de-embedded in the presented data. Operated at a $2 \mathrm{~V}$ supply voltage, the fabricated frontend consumes a dc current of $8 \mathrm{~mA}$. Fig. 6 shows the measured input reflection coefficient as the tunable load is adjusted for an RF of $4 \mathrm{GHz}$. As the $L C$ load is tuned for band selection, the input reflection coefficient varies negligibly due to the use of the cascode stage. It is apparent that a return loss better than $10 \mathrm{~dB}$ is achieved within 3 to $5 \mathrm{GHz}$. For the test of the conversion gain, differential LO signals are provided by a signal generator with an off-chip hybrid and phase shifters, while the output power of the differential IF signal is extracted through an off-chip power combiner.

For an IF frequency of $10 \mathrm{MHz}$, the down-conversion gain was characterized by sweeping the LO power. At various RF frequencies, the fabricated circuit exhibits a peak conversion gain with a LO power level in the vicinity of $3 \mathrm{dBm}$. As a fixed $\mathrm{LO}$ power of $3 \mathrm{dBm}$ is provided, Fig. 7 shows the conversion gain versus the IF frequency for various RF frequencies. The measured $-3 \mathrm{~dB}$ IF bandwidth ranges from 10 to $55 \mathrm{MHz}$. To evaluate the linearity of this circuit, a two-tone intermodulation distortion test was performed. The measurement results indicate a third-order intercept point (IIP3) of $-6 \mathrm{dBm}$ at the $4 \mathrm{GHz}$ band. In addition, the noise figure of the frontend receiver was also characterized. The measured single-sideband (SSB) noise figure is less than $17 \mathrm{~dB}$ within the entire frequency range. Table I sum-

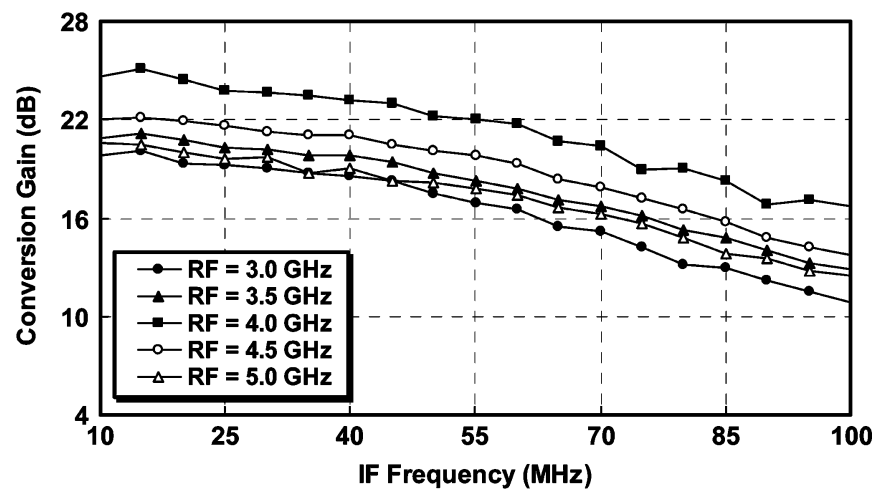

Fig. 7. Measured conversion gain versus the IF frequency.

TABLE I

Performance Summary of THE RECEIVER Front END

\begin{tabular}{|c|c|c|c|c|c|}
\hline Frequency $(\mathrm{GHz})$ & 3.0 & 3.5 & 4.0 & 4.5 & 5.0 \\
\hline Gain $(\mathrm{dB})$ & 19.8 & 20.9 & 24.6 & 22.0 & 20.6 \\
\hline SSB NF (dB) & 17.4 & 16.8 & 15.4 & 15.8 & 16.2 \\
\hline $\mathrm{P}_{\text {in-1dB }}(\mathrm{dBm})$ & -12.0 & -13.0 & -15.5 & -14.6 & -14.0 \\
\hline IIP3 $(\mathrm{dBm})$ & -2.4 & -5.5 & -6.0 & -3.0 & -2.7 \\
\hline $\begin{array}{c}\text { LO-to-IF } \\
\text { Isolation }(\mathrm{dB})\end{array}$ & 55.5 & 50.6 & 52.2 & 58.1 & 63.9 \\
\hline $\mathrm{S}_{11} \mid(\mathrm{dB})$ & -10.5 & -10.5 & -11.8 & -15.1 & -15.2 \\
\hline
\end{tabular}

marizes the performance of the fabricated frontend at various operating frequency bands.

\section{CONCLUSION}

A novel receiver frontend suitable for multiband wireless applications is demonstrated in this work. With the tunable $L C$ load and the broadband circuit techniques, a continuous tuning capability of the center frequency from 3 to $5 \mathrm{GHz}$ is achieved by the fully integrated frontend circuit implemented in a $0.18 \mu \mathrm{m}$ CMOS process.

\section{REFERENCES}

[1] C. F. Jou, K.-H. Cheng, W.-C. Lien, C.-H. Wu, and C.-H. Yen, "Design of a concurrent dual-band receiver front-end in 0.18- $\mu \mathrm{m}$ CMOS for WLANs IEEE 802.11 a/b/g applications," in Proc. IEEE Midwest Symp. Circuits Syst., Jul. 2004, vol. 1, pp. 177-180.

[2] A. Liscidini, M. Brandolini, D. Sanzogni, and R. Castello, "A 0.13$\mu \mathrm{m}$ CMOS front-end for DCS1800/UMTS/802.11b-g with multiband positive feedback low noise amplifier," in IEEE Symp. VLSI Circuit Dig., Jun. 2005, pp. 406-409.

[3] S.-W. Chung, S.-Y. Lee, and K.-H. Park, "Wideband impedance matching of integrated antennas and CMOS low noise amplifiers for a multiband UWB receiver," in Proc. IEEE Radio Wireless Conf., Sep. 2004, pp. 131-134.

[4] S.-G. Lee and J.-K. Choi, "Current-reuse bleeding mixer," Electron. Lett., vol. 36, no. 8, pp. 696-697, Apr. 2000.

[5] B. Razavi, Design of Integrated Circuits for Optical Communications. New York: McGraw-Hill, 2003.

[6] B. Analui and A. Hajimiri, "Bandwidth enhancement for transimpedance amplifiers," IEEE J. Solid-State Circuits, vol. 39, no. 8, pp. 1263-1270, Aug. 2004. 